Journal of the Egyptian Society of Parasitology, Vol.43, No.1, April 2013

J. Egypt. Soc. Parasitol., 43(1), 2013: 215 - 234

\title{
PHENOLIC CONTENT, ANTIOXIDANT POTENTIAL AND AEDES AEGYPTII ECOLOGICAL FRIEND LARVICIDAL ACTIVITY OF SOME SELECTED EGYPTIAN PLANTS \\ By
}

ATEF A. EL-HELA ${ }^{1}$, NEVEIN M. ABDEL-HADY ${ }^{2}$, GOUDA T.M.

DAWOUD $^{3}$, ABDO, M. HAMED ${ }^{4}$ AND TOSSON A. MORSY ${ }^{5}$

Department of Pharmacognosy ${ }^{1}$, Faculty of Pharmacy (Boys), Department of Pharmacognosy ${ }^{2}$, Faculty of Pharmacy (Girls), Department of Phytochemistry $^{3}$, Medicinal Plant Center, NODCAR, Department of Botany ${ }^{4}$, Faculty of Science, Al-Azhar University ${ }^{1,2.3}$ and Department of Parasitolo-

$\mathrm{gy}^{5}$, Faculty of Medicine, Ain-Shams University, Cairo 11566, Egypt

\begin{abstract}
Polyphenols constitute a distinct group of natural compounds of medicinal importance exhibiting wide range of physiological activities as antioxidant, immunestimulant, antitumor and antiparasitic. Yellow fever and dengue fever are mosquito-borne infectious diseases transmitted by Aedes aegyptii, the presence of yellow fever in Sudan and dengue fever in Saudi Arabia are threats to Egypt with the reemerging of Ae. aegyptii in Southern Egypt, larvae control is feasible than flying adults. This work was conducted targeting estimation of the relative levels of total phenolic content, antioxidant potential and larvicidal activity of 110 selected Egyptian plants. The highest total phenolic contents were estimated in aqueous extracts of Coronilla scorpioides L., Forsskaolea tenacissima L., Crataegus sinaica Boiss., Pistacia khinjuk Boiss. and Loranthus acacia Benth.; they were 916.70 \pm 4.80 , $813.70 \pm 4.16,744.90 \pm 4.93,549.00 \pm 3.93 \& 460.80 \pm 4.02 \mathrm{mg} \%$ while those of methanol extracts were estimated in Coronilla scorpioides, Forsskaolea tenacissima, Crataegus sinaica, Loranthus acacia and Pistacia khinjuk; they were 915.60 \pm 4.86 , $664.60 \pm 4.16,659.30 \pm 4.80,590.80 \pm 4.49 \& 588.00 \pm 3.85 \mathrm{mg} \%$ respectively. Investigation of the antioxidant potentials revealed that the most potent plants were Coronilla scorpioides, Forsskaolea tenacissima, Crataegus sinaica, Pistacia khinjuk and Loranthus acacia with calculated values of $454.80 \pm 4.83,418.4 \pm 4.16,399.10$ $\pm 4.90,342.5 \pm 2.72 \& 239.7 \pm 2.91 \%$ for aqueous extracts and $452.9 \pm 4.94,389.6$ $\pm 4.6,378.48 \pm 3.84,352.3 \pm 3.06 \& 346.5 \pm 2.98 \%$ for methanol extracts respectively while screening of larvicidal activity proved that Coronilla scorpioides, Forsskaolea tenacissima, Crataegus sinaica, Pistacia khinjuk and Loranthus acacia exhibited highest potency calculated as $22.53 \pm 2.01,23.85 \pm 2.07,28.17 \pm 2.06,31.60 \pm 2.93$ $\& 39.73 \pm 4.58 \mathrm{mg} \%$ aqueous extracts and $18.53 \pm 1.95,18.8 \pm 1.67,20.17 \pm 1.85$, $23.28 \pm 2.7 \& 28.48 \pm 3.9 \mathrm{mg} \%$ methanol ones respectively.
\end{abstract}

Keywords: Egypt, Antioxidants, Phenolic content, Aedes aegyptii, Yellow fever, Dengue virus, Larval control. 


\section{Introduction}

Natural products are derived from the phenomenon of biodiversity in which the interactions among organisms and their environment formulate these diverse complex chemical entities within the organisms that enhance their survival and competitiveness (Nurmikko et al, 2007), they are the main source for the majority of FDA-approved agents and are continued to be one of the major sources of inspiration for future drug discovery (Bhuwan et al, 2011). Phenolic compounds (phenolic acids, flavonoids, quinones, coumarins, lignans, tannins) are one of the main free radical scavenging molecules in plants (Cai et al, 2003; Zheng and Wang, 2001). Epidemiological studies have shown that many of these compounds possess anti-inflammatory, antiatherosclerotic, antitumor, antimutagenic, anticarcinogenic, antibacterial and antiviral activities (Owen et al, 2000; Sala et al, 2002; Cushnie and Lamb, 2005; El-Hela et al, 2011). Numerous physiological and biochemical processes in human body may produce oxygen entered free radicals and other reactive oxygen species as by-products, the overproduction of such free radicals can cause oxidative damage to biomolecules as lipids, proteins and even DNA, eventually leading to many chronic diseases, such as atherosclerosis, cancer, diabetes, aging, and other degenerative diseases in humans (Halliwell, 1994; Niki, 1997; Poulson et al, 1998).

The available synthetic antioxidants have been suspected of causing or prompting negative health effects, so strong restrictions encountered their application and there is an urgent trend to substitute them with naturally occurring antioxidants (Chu, 2000; Hosny and Rosazza 2002; Molyneux, 2004) while the intake of natural antioxidants has been associated with reduced risks of cancer, cardiovascular disease, diabetes, and other diseases associated with age which have the advantage of being almost devoid of side effects (Yang et al, 2001; Sun et al, 2002).

Yellow fever is endemic in 34 countries of Africa with a combined population of 468 Million (WHO 1996). Dengue fever and dengue hemorrhagic fever are vector-borne diseases of public health importance in tropical, subtropical, and temperate regions of the world infecting millions of people annually (Gubler 1998; Jacobs 2000; Pancharoen et al, 2000), dengue vaccine is not available, and the only effective vector intervention involves well organized larval control measures (Swawudhipong et al, 1992; Gratz, 1993; Kantachuvessiri, 2002; Pancharoen et al, 2002).

The principal vector of dengue and yellow viruses is Aedes aegyptii L. (Stegomia), Diptera: Culicidae, whose eggs are resistant to desiccation as they remain quiescent during the dry seasons and hatch only when rain fills breeding places (Abdal-magid and Alhusein 2008; Husham et al, 2010).

The use of synthetic organic insecticides in larvae control around the world has resulted in damage to the environment, pest resurgence and toxic effects on non-target organisms (Ab- 
udulai et al, 2001), in addition extensive use of chemical insecticides has made strains of the target insects resistant to most of them (Schaafsma et $a l, 1990)$ and so attention being diverted in favour of non-chemical methods for insect management.

More than 2,000 species of plants are known to have insecticidal properties (Klocke, 1989) while others have reported the bioactivity of extracts and essential oils from various plants against agricultural pests (Nagpal et al, 1996; Abdel-Hady et al 2005) and fortunately the plant derived insecticides encompasses an array of chemical compounds thus, the chance of insects developing resistance to such insecticides are less and also they are considered as ideal safe ecological friend insect controllers.

This perspective study aimed to the estimation of the total phenolic contents of the aqueous and methanol extracts of the 110 selected Egyptian plants to determine their antioxidant potentials as natural antioxidant drugs, and screening of the larvicidal activity of Ae. aegyptii targeting the discovery of natural ecological friend, costeffective alternative to the harmful chemical insecticides.

\section{Material, Equipment and Methods}

Plant material: The complete range of wild plant samples were collected at the flowering stage throughout Egypt, at random, from each of the five biogeographic Egyptian regions (Saint Catherine, South Sinai, 2008; Giza Zoo Garden, 2006; Garden of Faculty of Agriculture, Al-Azhar University, Nasr
City, 2006; River Nile, 2007 and gardens of the National Gene Bank, the National Institute of Horticulture, Faculty of Agriculture, Cairo University, 2008). The plant samples were identified taxonomically by Dr. Mohamed Tantawy, Prof. of Botany, Faculty of Science, Ain-Shams University and Dr. Moneer Abd El-Ghany, Prof. of Plant Taxonomy, Faculty of Science, Cairo, University.

The collected samples were airdried, powdered and kept in clean tightly closed amber coloured glass containers in a dark place at low temperature.

Voucher specimens were kept in the Herbarium Museum, Department of Pharmacognosy, Faculty of Pharmacy, Al-Azhar University.

Material for determination of total phenolic content: Folin-Ciocalteu's reagent (Sigma Chemical Co., St. Louis, MO, USA), and Gallic acid (E. Merck, Darmstadt, Germany).

Material for determination of antioxidant effect: DPPH (Sigma-Aldrich Quimica South Madrid Spain), Silica gel 60-F254 (Merck, Darmstadt, Germany), Mobile phase [butanol: acetic acid: water (40:10:50)] and Butylated hydroxyl toluene (BHT): SigmaAldrich Quimica South Madrid Spain.

Material for determination of larvicidal effect: egg rafts of Aedes aegyptii were obtained from Aswan and Tosh$\mathrm{ka}$, they were provided by last author.

Equipment: Soxhlet, Chromatographic glass jars, Rotatory evaporator (BUCHI Rotavapor ${ }^{\circledR}$ R-210/R-215, Germany), 96 Micro-well ${ }^{\mathrm{TM}}$ Plates, Conical 
Wells, Thermo Fisher Scientific USA, Genesys Spectrophotometer (Milton Roy, INC., Rochester, NY) for quantitative estimation of total phenolic content and Spectrophotometer (PerkinElmer Lambada 3) for quantitative determination of antioxidant effect.

Preparation of extracts: for the total methanol extract $50 \mathrm{~g}$ of each dried powdered plant under investigation was extracted separately by soxhlet for $24 \mathrm{~h}$ with methanol, after filtration, extracts were concentrated under vacuum then washed within hexane until the chlorophyll was completely removed; the washed methanol extracts were filtered and used for study while for the total aqueous extract $50 \mathrm{~g}$ of each powdered plant are infused in boiling distilled water set aside for $2 \mathrm{~h}$, filtered, after filtration, extracts were concentrated under vacuum, washed within hexane where the washed aqueous extracts were used for study.

Determination of the total phenolic content: The concentration of total phenolic compounds in the methanol extract of each plant was determined spectrophotometrically using the FolinCiocalteu's reagent which is a mixture of phosphomolybdate and phosphotungstate used for the colorimetric assay of phenolic compounds and polyphenol antioxidants to (Singleton and Rossi, 1965; McDonald et al, 2001). Standard curve was done using different concentrations of gallic acid (10:60 $\mathrm{mcg} / \mathrm{ml}$ ) in methanol, the concentrated extracts of the tested plants were dissolved each in least methanol volume then completed to $10 \mathrm{ml}, 100 \mu 1$ of each extract was separately diluted with 8 $\mathrm{ml}$ of distilled water. To each sample $0.5 \mathrm{ml}$ of $50 \%$ Folin-Ciocalteu's reagent were added, left $8 \mathrm{~min}$, and then $1.5 \mathrm{ml}$ of $5 \%$ sodium carbonate was added, mixed and allowed to stand for $60 \mathrm{~min}$. protected from light; their absorbance was measured at $725 \mathrm{~nm}$ using methanol as blank and the concentration of the total phenolic content of was calculated.

Determination of antioxidant potential: Determination of the antioxidant potential of each tested extract was done according to stable DPPH radical technique both qualitatively using thin layer chromatography (TLC) and quantitatively using the spectrophotometric method.

TLC assay: This was performed after Cavin et al. (1998) where $20 \mu$ l aliquot of the tested extract was spotted on silica gel plates and developed using butanol: acetic acid: water (40:10:50) as a mobile phase, after development, the dried TLC plates were sprayed with $0.2 \%$ DPPH solution in methanol and examined after $30 \mathrm{~min}$. active antioxidants compounds appeared as yellow spots against purple background.

Spectrophotometric assay: This was performed after Gialvez et al. (2005) where the test was carried out on 96 Micro-well plates. Standard curve was done using different concentrations of BHT (butylated hydroxytoluene) in methanol ( 7 serial 2 fold dilutions to give final range of 60 to $10 \mu \mathrm{g} / \mathrm{ml}$ ), $50 \mu 1$ of a $0.022 \%$ DPPH solution in methanol was added to a range solution of different concentrations ( 7 serial 3 fold solutions to give final range of 
1000 to $1.3 \mu \mathrm{g} / \mathrm{ml}$ of each extracts in methanol $(230 \mu \mathrm{l})$ and their absorbance were measured at $517 \mathrm{~nm}$ after $30 \mathrm{~min}$.

Determination of larvicidal activity: the egg rafts were reared in trays containing tape water and maintained at $28 \pm 2^{\circ} \mathrm{C}$ and when eggs were hatched into first instar larvae, they were fed with yeast powder and glucose, on the third day after hatching the first instar larvae moulted into second instar larvae and on the fifth day the third instar larvae were observed which moulted into fourth instar larvae the seventh day. The method of testing larvicidal action of the crude extracts was slightly modified from those of WHO (1996) where a stock solution was prepared by dissolving a known amount of the crude extract in an appropriate solvent and stored in a refrigerator at $15^{\circ} \mathrm{C}$. Twenty healthy, late $3^{\text {rd }}-4^{\text {th }}$ instar larvae were introduced into each testing cup (sterilized plastic drinking cup of $150 \mathrm{ml}$ capacity), which contained 100 $\mathrm{ml}$ of de-chlorinated tap water, a measured volume of stock solution was added to obtain the desired concentrations. Experiments were carried out with a series of five concentrations, each with three replicates, with a final total number of 60 larvae for each concentration; each batch of replicates contained one control of $100 \mathrm{ml}$ of tape-water and another of $100 \mathrm{ml}$ of water containing a volume of solvent corresponding to the maximum volume of extract tested.

As very few larvae succumbed within 24 hours of exposure to the test solutions, mortality was recorded after 48 hour of exposure, during which no food was offered to the larvae.

The mortalities of the larvae were recorded if moribund larvae were incapable of rising to the surface or of showing the characteristic diving reaction when the water was disturbed or they showed discoloration, unnatural position or rigor. The LC50 was determined by a Probit analysis program (Finney, 1971) and mortality was estimated by Abbott formula (1925).

Statistical analysis: The outcome data was carried out using one way analysis of variance (ANOVA) followed by student t-test, $\mathrm{P}$ value $<0.05$ were considered significant (Elliott and Woodward, 2007).

\section{Results}

The results are shown in table (1) and figures (1-4).

Fig. 1: Standard curve of gallic acid:

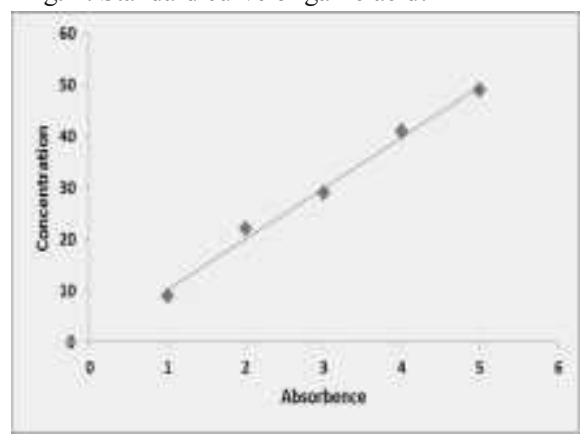

Fig. 2: Standard curve of butylated hydroxy toluene:

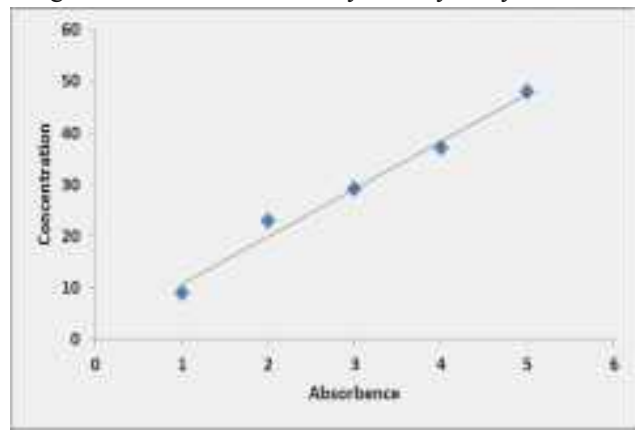


Table 1: Total phenolic content, antioxidant potential and larvicidal activity of the selected Egyptian plants:

\begin{tabular}{|c|c|c|c|c|c|c|}
\hline \multirow[b]{2}{*}{ Plant name } & \multicolumn{2}{|c|}{$\begin{array}{l}\text { Total Phenolic Content } \\
\text { (mg \%) }\end{array}$} & \multicolumn{2}{|c|}{$\begin{array}{l}\text { Antioxidant Potential } \\
\text { (\%Scavenging) }\end{array}$} & \multicolumn{2}{|c|}{$\begin{array}{l}\text { Larvicidal Activity } \\
\quad\left(\mathrm{LC}_{50} \mathrm{mg} \%\right)\end{array}$} \\
\hline & $\begin{array}{l}\text { Aqueous } \\
\text { extract }\end{array}$ & $\begin{array}{c}\text { Methanol } \\
\text { extract }\end{array}$ & $\begin{array}{l}\text { Aqueous } \\
\text { extract }\end{array}$ & $\begin{array}{c}\text { Methanol } \\
\text { extract }\end{array}$ & $\begin{array}{l}\text { Aqueous } \\
\text { extract }\end{array}$ & $\begin{array}{c}\text { Methanol } \\
\text { extract }\end{array}$ \\
\hline \multicolumn{7}{|l|}{ Acanthaceae } \\
\hline Blepharis ciliaris Boiss. & $73.10 \pm 2.70$ & $82.70 \pm 2.90$ & $28.10 \pm 2.03$ & $27.40 \pm 2.09$ & $106.50 \pm 3.61$ & $98.29 \pm 3.40$ \\
\hline \multicolumn{7}{|l|}{ Aizoaceae } \\
\hline Aizoon hispanicum L. & $48.30 \pm 1.93$ & $15.10 \pm 1.40$ & $25.00 \pm 2.63$ & $12.10 \pm 1.03$ & $113.82 \pm 3.70$ & $100.57 \pm 3.30$ \\
\hline Aizoon canariense L. & $38.10 \pm 1.50$ & $15.90 \pm 2.06$ & $24.40 \pm 2.82$ & $13.60 \pm 1.85$ & $134.40 \pm 4.64$ & $126.81 \pm 4.04$ \\
\hline \multicolumn{7}{|l|}{ Anacardiaceae } \\
\hline Pistacia khinjuk Boiss. & $549.00 \pm 3.93$ & $588.00 \pm 3.85$ & $342.50 \pm 2.72$ & $352.30 \pm 4.06$ & $31.60 \pm 2.93$ & $27.28 \pm 2.70$ \\
\hline \multicolumn{7}{|l|}{ Adiantaceae } \\
\hline Adiantum capillus veneris & $42.10 \pm 1.69$ & $49.00 \pm 2.06$ & $19.40 \pm 1.72$ & $21.30 \pm 1.90$ & $107.79 \pm 3.20$ & $99.26 \pm 3.30$ \\
\hline \multicolumn{7}{|l|}{ Apiaceae } \\
\hline Buplerum lancifolium & $78.60 \pm 2.82$ & $27.60 \pm 1.93$ & $27.80 \pm 2.04$ & $18.00 \pm 1.84$ & $58.95 \pm 3.20$ & $41.30 \pm 2.93$ \\
\hline Daucus syticus Murb. & $75.80 \pm 2.83$ & $49.00 \pm 2.72$ & $26.90 \pm 2.06$ & $19.70 \pm 1.29$ & $61.15 \pm 3.09$ & $53.77 \pm 3.20$ \\
\hline Ferula sinaicus Boiss. & $160.70 \pm 3.50$ & $86.70 \pm 3.06$ & $104.60 \pm 3.03$ & $88.40 \pm 2.92$ & $36.73 \pm 1.90$ & $19.59 \pm 1.99$ \\
\hline Malabaila suaveolens & $75.60 \pm 2.42$ & $23.60 \pm 2.30$ & $23.80 \pm 2.63$ & $16.00 \pm 1.79$ & $73.28 \pm 4.17$ & $68.00 \pm 4.35$ \\
\hline Scandex peten-veneris & $160.70 \pm 4.01$ & $86.70 \pm 3.20$ & $124.60 \pm 1.04$ & $98.40 \pm 2.05$ & $44.69 \pm 3.90$ & $31.60 \pm 2.92$ \\
\hline \multicolumn{7}{|l|}{ Asclepiadaceae } \\
\hline Calotropis procera Ait. & $90.20 \pm 3.31$ & $50.80 \pm 2.70$ & $29.40 \pm 2.15$ & $21.70 \pm 1.80$ & $67.40 \pm 3.39$ & $59.67 \pm 3.50$ \\
\hline Gomphocarpus sinaicus & $133.30 \pm 3.48$ & $63.90 \pm 2.02$ & $25.90 \pm 2.10$ & $23.80 \pm 2.00$ & $79.05 \pm 2.68$ & $68.17 \pm 2.07$ \\
\hline Cynanchum actum & $91.20 \pm 2.96$ & $53.80 \pm 1.53$ & $29.80 \pm 2.74$ & $21.90 \pm 1.26$ & $82.58 \pm 3.80$ & $77.50 \pm 3.62$ \\
\hline Caralluma sinainca & $79.00 \pm 5.09$ & $56.80 \pm 4.80$ & $27.40 \pm 1.95$ & $22.40 \pm 1.62$ & $83.40 \pm 3.99$ & $78.15 \pm 3.90$ \\
\hline \multicolumn{7}{|l|}{ Asteraceae } \\
\hline Achillea santolina $\mathrm{L}$. & $72.60 \pm 3.09$ & $60.20 \pm 2.70$ & $23.50 \pm 2.95$ & $19.2 \pm 2.60$ & $91.51 \pm 3.86$ & $84.90 \pm 3.60$ \\
\hline Ageratum conyzoides & $56.9 \pm 2.14$ & $51.20 \pm 2.09$ & $18.70 \pm 1.96$ & $17.10 \pm 1.63$ & $58.40 \pm 3.90$ & $44.28 \pm 3.30$ \\
\hline Amberboa leucantha & $76.70 \pm 3.02$ & $85.30 \pm 2.97$ & $33.90 \pm 2.80$ & $20.50 \pm 1.90$ & $55.84 \pm 3.30$ & $49.71 \pm 3.20$ \\
\hline Anacyclus alexanderinus & $63.60 \pm 2.62$ & $53.20 \pm 2.90$ & $19.70 \pm 2.30$ & $18.50 \pm 1.90$ & $49.39 \pm 3.50$ & $40.16 \pm 2.80$ \\
\hline Astriscus graveolens & $55.80 \pm 2.90$ & $65.30 \pm 2.04$ & $20.20 \pm 2.83$ & $19.60 \pm 2.90$ & $68.20 \pm 2.50$ & $56.08 \pm 3.36$ \\
\hline Carduus getulus & $189.00 \pm 3.94$ & $214.90 \pm 3.73$ & $109.10 \pm 3.86$ & $122.10 \pm 3.70$ & $86.94 \pm 3.02$ & $80.50 \pm 2.90$ \\
\hline Carthamus tenuis & $162.30 \pm 9.03$ & $61.80 \pm 2.10$ & $107.30 \pm 2.84$ & $76.40 \pm 2.51$ & $100.92 \pm 3.28$ & $94.60 \pm 3.69$ \\
\hline Centaurea ammocy- & $85.00 \pm 2.05$ & $50.40 \pm 1.79$ & $18.20 \pm 1.92$ & $16.90 \pm 1.80$ & $68.58 \pm 3.50$ & $55.40 \pm 3.65$ \\
\hline Dittrichia viscosa & $269.80 \pm 3.30$ & $247.80 \pm 2.82$ & $107.30 \pm 2.70$ & $103.90 \pm 2.03$ & $59.40 \pm 3.20$ & $60.29 \pm 3.03$ \\
\hline Echinops hussoni & $100.30 \pm 3.07$ & $77.80 \pm 2.75$ & $34.50 \pm 2.01$ & $19.60 \pm 2.30$ & $95.96 \pm 4.30$ & $88.50 \pm 3.96$ \\
\hline Helichrysum conglobatum & $232.30 \pm 4.09$ & $164.80 \pm 3.20$ & $111.30 \pm 2.80$ & $104.70 \pm 2.99$ & $59.05 \pm 4.80$ & $60.03 \pm 3.88$ \\
\hline Iphiona mucronata & $65.20 \pm 2.04$ & $46.20 \pm 2.00$ & $21.30 \pm 2.74$ & $1790 \pm 2.66$ & $110.39 \pm 4.18$ & $96.40 \pm 3.60$ \\
\hline Matricaria recutita $\mathrm{L}$. & $147.60 \pm 3.03$ & $142.40 \pm 2.14$ & $45.90 \pm 2.80$ & $43.30 \pm 1.88$ & $58.69 \pm 3.05$ & $48.20 \pm 2.90$ \\
\hline Notobasis syriaca $\mathrm{L}$. & $64.00 \pm 1.85$ & $43.80 \pm 1.99$ & $13.70 \pm 1.53$ & $11.70 \pm 1.15$ & $64.10 \pm 3.96$ & $55.30 \pm 3.94$ \\
\hline Pallenis spinosa $\mathrm{L}$. Cass. & $55.70 \pm 1.70$ & $57.10 \pm 1.20$ & $13.60 \pm 2.00$ & $12.80 \pm 1.69$ & $88.44 \pm 4.60$ & $78.30 \pm 4.75$ \\
\hline \multicolumn{7}{|l|}{ Boraginaceae } \\
\hline Alkanna tinctoria $\mathrm{L}$. & $157.90 \pm 3.37$ & $104.20 \pm 2.74$ & $116.70 \pm 3.11$ & $101.30 \pm 3.22$ & $109.20 \pm 3.80$ & $99.60 \pm 3.90$ \\
\hline Anchusa azurea Mill. & $83.3 \pm 2.80$ & $88.20 \pm 2.27$ & $22.30 \pm 2.74$ & $26.70 \pm 2.25$ & $120.38 \pm 3.06$ & $113.59 \pm 3.27$ \\
\hline Anchusa aegypt & $66.7 \pm 2.90$ & $43.60 \pm 2.39$ & $19.90 \pm 2.80$ & $16.40 \pm 1.70$ & $107.50 \pm 3.33$ & $98.21 \pm 3.95$ \\
\hline Asperugo procu & $189.80 \pm 7.82$ & $87.50 \pm 4.79$ & $96.00 \pm 2.74$ & $75.70 \pm 2.06$ & $84.37 \pm 3.26$ & $80.50 \pm 3.03$ \\
\hline Echium lycopsis L. & $148.90 \pm 3.02$ & $108.20 \pm 2.95$ & $82.90 \pm 2.64$ & $41.20 \pm 2.55$ & $126.49 \pm 4.11$ & $118.57 \pm 4.09$ \\
\hline Echium setosum Vahl. & $70.20 \pm 3.09$ & $71.30 \pm 3.21$ & $21.70 \pm 1.70$ & $22.50 \pm 1.20$ & $117.39 \pm 3.20$ & $108.30 \pm 3.72$ \\
\hline Lappula spinoca & $227.50 \pm 6.18$ & $124.30 \pm 5.88$ & $134.90 \pm 2.70$ & $96.80 \pm 2.53$ & $88.07 \pm 3.30$ & $78.40 \pm 3.02$ \\
\hline Paracaryum rugulosum- & $140.60 \pm 6.83$ & $98.00 \pm 4.55$ & $52.70 \pm 2.46$ & $45.30 \pm 2.57$ & $95.79 \pm 3.91$ & $87.90 \pm 3.05$ \\
\hline \multicolumn{7}{|l|}{ Brassicaceae } \\
\hline Eruca sativa Miller & $64.60 \pm 2.60$ & $34.60 \pm 2.11$ & $29.00 \pm 2.90$ & $19.80 \pm 1.89$ & $102.47 \pm 3.20$ & $91.30 \pm 3.90$ \\
\hline
\end{tabular}




\begin{tabular}{|c|c|c|c|c|c|c|}
\hline \multirow[b]{2}{*}{ Plant name } & \multicolumn{2}{|c|}{$\begin{array}{c}\text { Total Phenolic Content } \\
\text { (mg \%) }\end{array}$} & \multicolumn{2}{|c|}{$\begin{array}{l}\text { Antioxidant Potential } \\
\text { (\%Scavenging) }\end{array}$} & \multicolumn{2}{|c|}{$\begin{array}{l}\text { Larvicidal Activity } \\
\left(\mathrm{LC}_{50} \mathrm{mg} \%\right)\end{array}$} \\
\hline & $\begin{array}{c}\text { Aqueous } \\
\text { extract }\end{array}$ & $\begin{array}{c}\text { Methanol } \\
\text { extract }\end{array}$ & $\begin{array}{c}\text { Aqueous } \\
\text { extract }\end{array}$ & $\begin{array}{c}\text { Methanol } \\
\text { extract }\end{array}$ & $\begin{array}{c}\text { Aqueous } \\
\text { extract }\end{array}$ & $\begin{array}{c}\text { Methanol } \\
\text { extract }\end{array}$ \\
\hline Farsetia aegyptia L. & $49.70 \pm 1.42$ & $26.30 \pm 1.10$ & $17.80 \pm 0.99$ & $18.50 \pm 0.76$ & $165.40 \pm 4.38$ & $158.32 \pm 4.79$ \\
\hline Zilla spinosa L. Prantl & $42.90 \pm 2.80$ & $23.80 \pm 2.30$ & $17.50 \pm 0.81$ & $14.90 \pm 0.55$ & $176.49 \pm 4.71$ & $160.48 \pm 4.30$ \\
\hline \multicolumn{7}{|l|}{ Caesalpiniaceae } \\
\hline Cassia italica Mill. & $79.00 \pm 3.30$ & $53.70 \pm 2.99$ & $34.50 \pm 2.30$ & $29.30 \pm 1.91$ & $143.72 \pm 4.29$ & $130.50 \pm 4.80$ \\
\hline \multicolumn{7}{|l|}{ Capparaceae } \\
\hline Capparis aegyptia L. & $91.80 \pm 3.88$ & $81.60 \pm 3.29$ & $28.30 \pm 2.10$ & $26.3 \pm 1.94$ & $197.10 \pm 4.22$ & $186.47 \pm 3.90$ \\
\hline \multicolumn{7}{|l|}{ Chinopodiaceae } \\
\hline Hammada eigii lljin & $49.30 \pm 2.60$ & $30.60 \pm 1.97$ & $18.30 \pm 1.97$ & $14.80 \pm 1.70$ & $55.86 \pm 3.59$ & $46.01 \pm 2.70$ \\
\hline Salsola kali L. & $58.40 \pm 2.90$ & $34.80 \pm 2.20$ & $19.90 \pm 1.29$ & $17.30 \pm 1.00$ & $58.28 \pm 3.64$ & $46.50 \pm 2.94$ \\
\hline Salsola volkensii Asch. & $41.30 \pm 2.30$ & $27.50 \pm 2.10$ & $17.40 \pm 0.95$ & $15.50 \pm 0.80$ & $79.57 \pm 2.30$ & $66.05 \pm 2.49$ \\
\hline Salsola vermiculata $\mathrm{L}$. & $40.80 \pm 2.64$ & $32.60 \pm 2.14$ & $17.90 \pm 1.07$ & $17.50 \pm 0.90$ & $50.37 \pm 3.50$ & $41.93 \pm 2.49$ \\
\hline \multicolumn{7}{|l|}{ Cistaceae } \\
\hline Helianthemum cilliatum & $274.20 \pm 3.40$ & $176.10 \pm 3.27$ & $188.50 \pm 3.45$ & $125.80 \pm 2.39$ & $117.10 \pm 3.30$ & $102.40 \pm 2.77$ \\
\hline Helianthemum lodifolium & $227.80 \pm 3.26$ & $155.60 \pm 2.80$ & $169.40 \pm 3.21$ & $121.30 \pm 2.17$ & $148.47 \pm 3.80$ & $130.15 \pm 3.05$ \\
\hline Helianthemum sphaero- & $141.80 \pm 2.99$ & $74.90 \pm 2.07$ & $58.50 \pm 2.89$ & $38.80 \pm 2.50$ & $139.48 \pm 3.40$ & $129.11 \pm 3.04$ \\
\hline \multicolumn{7}{|l|}{$\stackrel{1}{\text { Cucubitaceae }}$} \\
\hline Bryonia syriaca Bioss. & $120.80 \pm 3.64$ & $65.70 \pm 3.00$ & $48.40 \pm 2.10$ & $19.80 \pm 1.63$ & $115.29 \pm 3.19$ & $99.20 \pm 2.87$ \\
\hline Bryonia cretica $\mathrm{L}$. & $170.50 \pm 5.74$ & $22.90 \pm 2.47$ & $59.60 \pm 2.90$ & $11.60 \pm 0.96$ & $130.20 \pm 3.28$ & $118.27 \pm 3.26$ \\
\hline Cucumus prophetarum & $141.70 \pm 3.80$ & $79.80 \pm 3.15$ & $58.90 \pm 2.90$ & $41.50 \pm 2.60$ & $143.55 \pm 4.09$ & $137.74 \pm 4.95$ \\
\hline \multicolumn{7}{|l|}{ Cycadaceae } \\
\hline Cycas beddomei & $218.40 \pm 3.30$ & $237.50 \pm 3.07$ & $133.80 \pm 2.60$ & $141.69 \pm 2.49$ & $148.39 \pm 4.39$ & $136.50 \pm 4.88$ \\
\hline Cycas circinalis & $197.49 \pm 3.38$ & $211.30 \pm 2.05$ & $127.85 \pm 2.39$ & $131.20 \pm 2.42$ & $133.80 \pm 4.26$ & $127.40 \pm 3.09$ \\
\hline Cycas revolute & $232.57 \pm 3.84$ & $247.90 \pm 3.30$ & $137.80 \pm 3.01$ & $142.95 \pm 2.96$ & $127.60 \pm 4.86$ & $116.69 \pm 4.70$ \\
\hline \multicolumn{7}{|l|}{ Dipsacaceae } \\
\hline Petrocephalus pappo- & $143.60 \pm 3.09$ & $78.50 \pm 2.96$ & $55.90 \pm 3.30$ & $37.60 \pm 2.90$ & $228.98 \pm 4.16$ & $224.60 \pm 3.36$ \\
\hline Petrocephalus sanctus & $86.50 \pm 2.60$ & $97.20 \pm 3.69$ & $39.60 \pm 3.11$ & $43.10 \pm 2.30$ & $184.40 \pm 3.09$ & $175.20 \pm 2.04$ \\
\hline \multicolumn{7}{|l|}{ Ephedraceae } \\
\hline Ephedra alata Decne & $47.50 \pm 2.44$ & $66.30 \pm 2.60$ & $18.80 \pm 1.88$ & $19.20 \pm 2.01$ & $153.80 \pm 4.58$ & $139.40 \pm 4.10$ \\
\hline Ephedra aphylla & $49.60 \pm 2.70$ & $68.70 \pm 3.05$ & $19.50 \pm 1.64$ & $20.00 \pm 2.50$ & $195.72 \pm 4.06$ & $182.30 \pm 4.90$ \\
\hline \multicolumn{7}{|l|}{ Eqiusetaceae } \\
\hline Eqiusetium ramossissinum & $39.10 \pm 2.07$ & $56.80 \pm 2.30$ & $19.50 \pm 3.36$ & $19.60 \pm 1.96$ & $185.46 \pm 3.61$ & $172.59 \pm 3.90$ \\
\hline \multicolumn{7}{|l|}{ Fabaceae } \\
\hline Coronilla scorpioides & $916.70 \pm 4.80$ & $915.60 \pm 4.86$ & $354.80 \pm 4.03$ & $352.90 \pm 4.94$ & $27.53 \pm 2.01$ & $18.53 \pm 1.95$ \\
\hline Lathyrus aphaca $\mathrm{L}$. & $44.20 \pm 2.40$ & $60.50 \pm 2.15$ & $11.30 \pm 1.83$ & $18.60 \pm 2.07$ & $145.20 \pm 3.90$ & $138.40 \pm 3.37$ \\
\hline Melilotus sulcata Desf. & $60.30 \pm 2.46$ & $298.7 \pm 3.90$ & $42.60 \pm 3.11$ & $105.40 \pm 4.03$ & $141.40 \pm 3.79$ & $133.50 \pm 3.05$ \\
\hline Ononis veginalis Vahl. & $48.40 \pm 2.57$ & $64.60 \pm 3.04$ & $14.80 \pm 1.50$ & $18.30 \pm 1.92$ & $188.20 \pm 4.58$ & $179.28 \pm 3.90$ \\
\hline Ononis serrata Forssk & $85.90 \pm 3.94$ & $47.80 \pm 3.11$ & $21.00 \pm 1.80$ & $13.60 \pm 1.25$ & $190.16 \pm 4.27$ & $177.26 \pm 3.85$ \\
\hline Retama raetam Forssk & $133.30 \pm 4.01$ & $67.90 \pm 2.60$ & $29.50 \pm 1.99$ & $24.30 \pm 1.75$ & $173.10 \pm 4.05$ & $166.30 \pm 3.81$ \\
\hline \multicolumn{7}{|l|}{ Geraniaceae } \\
\hline Erodium bryoniifolium & $45.40 \pm 2.10$ & $25.40 \pm 2.07$ & $20.10 \pm 1.83$ & $11.90 \pm 1.79$ & $67.90 \pm 3.69$ & $55.38 \pm 3.09$ \\
\hline Monsonia nivea Dence & $45.70 \pm 2.70$ & $25.90 \pm 2.38$ & $19.30 \pm 1.74$ & $12.80 \pm 1.92$ & $44.92 \pm 2.95$ & $39.75 \pm 2.80$ \\
\hline \multicolumn{7}{|l|}{ Ginkgoaceae } \\
\hline Ginkgo biloba L.C. & $321.90 \pm 4.29$ & $278.60 \pm 3.30$ & $189.50 \pm 2.94$ & $185.60 \pm 2.70$ & $57.60 \pm 3.58$ & $68.90 \pm 3.19$ \\
\hline \multicolumn{7}{|l|}{ Hypericaceae } \\
\hline Hypericum sinaicum & $440.60 \pm 3.80$ & $535.80 \pm 4.23$ & $244.60 \pm 3.00$ & $257.20 \pm 2.63$ & $79.54 \pm 3.70$ & $68.27 \pm 3.29$ \\
\hline \multicolumn{7}{|l|}{ Lamiaceae } \\
\hline Eremostachys laciniata & $85.60 \pm 2.05$ & $89.40 \pm 2.06$ & $19.20 \pm 1.93$ & $19.40 \pm 2.00$ & $77.42 \pm 3.90$ & $71.38 \pm 3.85$ \\
\hline Ballota kaiseri Tackh. & $90.60 \pm 3.43$ & $74.80 \pm 3.11$ & $25.60 \pm 2.94$ & $21.90 \pm 3.29$ & $89.18 \pm 4.03$ & $80.59 \pm 3.97$ \\
\hline
\end{tabular}




\begin{tabular}{|c|c|c|c|c|c|c|}
\hline \multirow[b]{2}{*}{ Plant name } & \multicolumn{2}{|c|}{$\begin{array}{c}\text { Total Phenolic Content } \\
(\mathrm{mg} \%)\end{array}$} & \multicolumn{2}{|c|}{$\begin{array}{l}\text { Antioxidant Potential } \\
\text { (\%Scavenging) }\end{array}$} & \multicolumn{2}{|c|}{$\begin{array}{l}\text { Larvicidal Activity } \\
\left(\text { LC }_{50} \mathrm{mg} \%\right)\end{array}$} \\
\hline & $\begin{array}{c}\text { Aqueous } \\
\text { extract }\end{array}$ & $\begin{array}{c}\text { Methanol } \\
\text { extract }\end{array}$ & $\begin{array}{c}\text { Aqueous } \\
\text { extract }\end{array}$ & $\begin{array}{c}\text { Methanol } \\
\text { extract }\end{array}$ & $\begin{array}{c}\text { Aqueous } \\
\text { extract }\end{array}$ & $\begin{array}{c}\text { Methanol } \\
\text { extract }\end{array}$ \\
\hline Marrubium alysson L. & $76.70 \pm 2.90$ & $53.20 \pm 2.63$ & $24.10 \pm 1.50$ & $21.80 \pm 1.95$ & $76.60 \pm 3.37$ & $67.61 \pm 3.05$ \\
\hline Phlomis aurea Decne & $59.60 \pm 2.66$ & $60.50 \pm 2.91$ & $15.70 \pm 1.00$ & $16.20 \pm 1.41$ & $86.11 \pm 4.02$ & $79.51 \pm 3.58$ \\
\hline Phlomis floccose $\mathrm{D}$. & $54.20 \pm 2.40$ & $46.90 \pm 2.10$ & $14.80 \pm 0.91$ & $13.60 \pm 1.18$ & $99.61 \pm 4.30$ & $87.35 \pm 4.20$ \\
\hline Salvia aegyptiaca $\mathrm{L}$. & $29.50 \pm 2.04$ & $30.30 \pm 2.17$ & $16.70 \pm 1.26$ & $16.20 \pm 1.03$ & $100.82 \pm 4.40$ & $92.66 \pm 4.50$ \\
\hline Salvia lanigera Poir. & $223.90 \pm 3.13$ & $155.80 \pm 2.50$ & $133.90 \pm 2.70$ & $124.70 \pm 2.03$ & $75.00 \pm 3.30$ & $67.10 \pm 3.04$ \\
\hline Salvia spinosa L.D. & $90.60 \pm 3.05$ & $74.80 \pm 2.99$ & $23.60 \pm 2.30$ & $21.90 \pm 2.38$ & $98.69 \pm 4.28$ & $86.4 \pm 3.90$ \\
\hline Lamium amplexiccaula & $85.00 \pm 2.60$ & $77.50 \pm 2.49$ & $22.80 \pm 2.00$ & $21.40 \pm 1.30$ & $107.64 \pm 4.50$ & $88.84 \pm 3.27$ \\
\hline \multicolumn{7}{|l|}{ Liliacaeae } \\
\hline Asparagus aphyllus $\mathrm{L}$. & $65.30 \pm 2.09$ & $37.80 \pm 1.74$ & $15.60 \pm 2.17$ & $9.70 \pm 0.93$ & $105.93 \pm 3.07$ & $102.00 \pm 2.85$ \\
\hline Asparagus stipularis & $61.20 \pm 2.03$ & $39.60 \pm 1.80$ & $17.60 \pm 1.93$ & $13.20 \pm 1.06$ & $104.52 \pm 3.70$ & $96.30 \pm 3.38$ \\
\hline Scilla hanburyi Baker. & $81.40 \pm 2.39$ & $44.70 \pm 2.05$ & $21.20 \pm 1.96$ & $19.66 \pm 0.82$ & $102.68 \pm 3.09$ & $99.70 \pm 2.95$ \\
\hline \multicolumn{7}{|l|}{ Loranthaceae } \\
\hline Loranthus acacia & $460.80 \pm 4.02$ & $590.80 \pm 4.49$ & $239.70 \pm 2.91$ & $246.50 \pm 2.98$ & $28.73 \pm 2.58$ & $19.48 \pm 1.90$ \\
\hline \multicolumn{7}{|l|}{ Malvaceae } \\
\hline Alcea striats (DC.) & $20.50 \pm 2.10$ & $24.40 \pm 1.97$ & $12.30 \pm 1.42$ & $5.70 \pm 0.74$ & $202.15 \pm 4.06$ & $191.10 \pm 3.56$ \\
\hline \multicolumn{7}{|l|}{ Papavaraceae } \\
\hline Argemone mexicana $\mathrm{L}$. & $65.30 \pm 2.89$ & $29.70 \pm 2.09$ & $19.30 \pm 1.84$ & $15.10 \pm 1.05$ & $153.52 \pm 5.48$ & $146.20 \pm 5.37$ \\
\hline Glaucium corniculatum & $161.90 \pm 3.90$ & $81.20 \pm 2.70$ & $120.60 \pm 3.15$ & $118.80 \pm 2.93$ & $127.40 \pm 4.47$ & $117.35 \pm 3.90$ \\
\hline Papver decaisnei & $77.80 \pm 3.01$ & $69.40 \pm 2.95$ & $26.40 \pm 1.70$ & $23.90 \pm 1.54$ & $150.18 \pm 4.62$ & $139.60 \pm 4.05$ \\
\hline Papver hybridum $\mathrm{L}$. & $70.50 \pm 3.16$ & $58.90 \pm 3.07$ & $24.60 \pm 1.83$ & $22.60 \pm 1.21$ & $147.36 \pm 5.86$ & $137.52 \pm 4.79$ \\
\hline \multicolumn{7}{|l|}{ Plantaginaceae } \\
\hline Plantago lanceolata & $27.70 \pm 2.06$ & $43.20 \pm 2.38$ & $6.60 \pm 0.94$ & $7.80 \pm 0.98$ & $145.94 \pm 5.50$ & $138.61 \pm 4.95$ \\
\hline Plantago sianica L. & $29.40 \pm 1.90$ & $45.60 \pm 2.39$ & $7.60 \pm 1.17$ & $9.00 \pm 1.05$ & $130.68 \pm 4.49$ & $119.58 \pm 3.63$ \\
\hline \multicolumn{7}{|l|}{ Polygonaceae } \\
\hline Rumex pictus Forssk & $161.20 \pm 3.70$ & $60.60 \pm 3.17$ & $121.30 \pm 2.95$ & $110.90 \pm 3.92$ & $139.26 \pm 5.38$ & $121.67 \pm 4.70$ \\
\hline \multicolumn{7}{|l|}{ Rununculaceae } \\
\hline Ranunculus sceleratus & $14.60 \pm 1.09$ & $16.80 \pm 1.10$ & $6.30 \pm 0.93$ & $8.40 \pm 1.03$ & $203.75 \pm 6.69$ & $192.50 \pm 5.90$ \\
\hline \multicolumn{7}{|l|}{ Resedaceae } \\
\hline Ochradenus baccatus & $79.20 \pm 3.22$ & $45.80 \pm 2.75$ & $23.70 \pm 2.03$ & $8.20 \pm 0.95$ & $176.90 \pm 7.70$ & $164.61 \pm 6.62$ \\
\hline \multicolumn{7}{|l|}{ Rhumnaceae } \\
\hline Ziziphus lotus L. Lam. & $238.20 \pm 3.20$ & $200.70 \pm 3.19$ & $135.60 \pm 3.95$ & $129.40 \pm 3.57$ & $148.37 \pm 5.59$ & $142.40 \pm 4.20$ \\
\hline \multicolumn{7}{|l|}{ Roseceae } \\
\hline Crataegus sinaica & $744.90 \pm 4.93$ & $659.30 \pm 4.80$ & $399.10 \pm 4.90$ & $378.48 \pm 3.84$ & $28.17 \pm 2.06$ & $21.17 \pm 1.85$ \\
\hline Potentilla supine L. & $140.50 \pm 3.40$ & $170.80 \pm 3.79$ & $22.50 \pm 2.30$ & $27.30 \pm 3.09$ & $69.58 \pm 3.49$ & $52.5 \pm 3.09$ \\
\hline \multicolumn{7}{|l|}{ Rutaceae } \\
\hline Ruta tuberculata For- & $83.60 \pm 3.18$ & $72.60 \pm 3.10$ & $21.10 \pm 2.39$ & $19.50 \pm 2.00$ & $108.15 \pm 4.69$ & $99.07 \pm 3.93$ \\
\hline \multicolumn{7}{|l|}{ Salvadoraceae } \\
\hline Salvadora persica $\mathrm{L}$. & $48.80 \pm 2.90$ & $42.50 \pm 2.73$ & $18.90 \pm 2.25$ & $15.30 \pm 1.98$ & $190.17 \pm 5.88$ & $180.28 \pm 5.20$ \\
\hline \multicolumn{7}{|l|}{ Scrophilareaceae } \\
\hline Kickxia aegyptiaca $\mathrm{L}$. & $45.20 \pm 2.04$ & $38.10 \pm 1.95$ & $16.70 \pm 1.96$ & $19.10 \pm 0.95$ & $110.46 \pm 3.30$ & $102.09 \pm 2.93$ \\
\hline Kickxia heterophylla & $49.70 \pm 3.09$ & $48.20 \pm 2.96$ & $18.90 \pm 2.28$ & $17.60 \pm 1,80$ & $104.06 \pm 3.40$ & $98.48 \pm 3.96$ \\
\hline Scrophularia arguta & $79.30 \pm 2.90$ & $50.10 \pm 2.73$ & $18.50 \pm 2.06$ & $12.90 \pm 1.70$ & $118.67 \pm 3.05$ & $108.22 \pm 3.58$ \\
\hline Verbascum fruticu- & $68.40 \pm 2.72$ & $46.20 \pm 2.18$ & $20.40 \pm 2.04$ & $17.50 \pm 2.10$ & $148.37 \pm 4.57$ & $136.80 \pm 4.61$ \\
\hline Veronica anagallis- & $38.00 \pm 2.30$ & $38.90 \pm 2.38$ & $15.40 \pm 1.03$ & $18.70 \pm 0.91$ & $109.56 \pm 4.20$ & $102.88 \pm 4.19$ \\
\hline \multicolumn{7}{|l|}{ Solanaceae } \\
\hline Hyoscyamus aureus $\mathrm{L}$. & $56.90 \pm 2.96$ & $51.80 \pm 2.50$ & $18.30 \pm 1.90$ & $19.10 \pm 2.01$ & $208.63 \pm 4.70$ & $201.80 \pm 4.28$ \\
\hline \multicolumn{7}{|l|}{ Verbenaceae } \\
\hline Verbena tenara & $198.50 \pm 3.10$ & $211.80 \pm 3.27$ & $95.30 \pm 2.36$ & $97.20 \pm 2.45$ & $73.95 \pm 4.50$ & $67.66 \pm 3.37$ \\
\hline
\end{tabular}




\begin{tabular}{|c|c|c|c|c|c|c|}
\hline \multicolumn{7}{|l|}{ Cont., } \\
\hline \multirow[b]{2}{*}{ Plant name } & \multicolumn{2}{|c|}{$\begin{array}{c}\text { Total Phenolic Content } \\
(\mathrm{mg} \%)\end{array}$} & \multicolumn{2}{|c|}{$\begin{array}{c}\text { Antioxidant Potential } \\
(\% \text { Scavenging })\end{array}$} & \multicolumn{2}{|c|}{$\begin{array}{l}\text { Larvicidal Activity } \\
\left(\mathrm{LC}_{50} \mathrm{mg} \%\right)\end{array}$} \\
\hline & $\begin{array}{l}\text { Aqueous } \\
\text { extract }\end{array}$ & $\begin{array}{c}\text { Methanol } \\
\text { extract }\end{array}$ & $\begin{array}{l}\text { Aqueous } \\
\text { extract }\end{array}$ & $\begin{array}{c}\text { Methanol } \\
\text { extract }\end{array}$ & $\begin{array}{c}\text { Aqueous } \\
\text { extract }\end{array}$ & $\begin{array}{c}\text { Methanol } \\
\text { extract }\end{array}$ \\
\hline Verbena rigda Hand & $143.30 \pm 2.85$ & $176.80 \pm 3.90$ & $96.40 \pm 2.84$ & $97.40 \pm 2.39$ & $84.62 \pm 4.60$ & $78.80 \pm 3.47$ \\
\hline Verbena venosa & $199.50 \pm 3.41$ & $185.90 \pm 3.50$ & $93.90 \pm 2.95$ & $90.50 \pm 2.85$ & $86.92 \pm 5.06$ & $90.30 \pm 4.95$ \\
\hline Lantana camara $\mathrm{L}$. & $249.38 \pm 3.48$ & $265.80 \pm 3.01$ & $106.40 \pm 3.60$ & $115.73 \pm 3.95$ & $49.68 \pm 1.84$ & $46.30 \pm 1.59$ \\
\hline \multicolumn{7}{|l|}{ Urtiaceae } \\
\hline Forsskaolea tenacissi- & $813.70 \pm 4.16$ & $664.60 \pm 3.70$ & $418.40 \pm 4.16$ & $389.60 \pm 4.60$ & $23.85 \pm 2.07$ & $18.85 \pm 1.67$ \\
\hline Urtica pilulifera $\mathrm{L}$. & $49.50 \pm 3.07$ & $20.60 \pm 2.11$ & $17.90 \pm 1.85$ & $16.20 \pm 0.60$ & $68.15 \pm 2.99$ & $60.28 \pm 2.90$ \\
\hline
\end{tabular}

Results $=$ mean of three measurements \pm standard error, Total phenolic content calculated as gallic acid equivalents GAE $\mathrm{mg} \%$, \% scavenging potential for Butyl hydroxyl toluene (BHT) "Antioxidant synthetic standard=93 \pm 0.50 at dose level of $0.4 \mathrm{mg} / \mathrm{ml}$, scavenging potential for Quercetin "Antioxidant natural standard=95.6 \pm 0.40 at dose level of $0.025 \mathrm{mg} / \mathrm{ml}$.

\section{Discussion}

Phenolic compounds is a generic term that refers to a large number of compounds $(<8,000)$ widely dispersed throughout the plant kingdom characterized by having at least one aromatic ring with one or more hydroxyl groups attached, they range from simple, low molecular-weight, the single aromaticringed compounds to large and complex tannins and derived polyphenols (Crozier et al, 2006; Pereira et al, 2009). Medicinal plant are commonly rich in phenolic compounds with many useful properties for human health as anti-inflammatory, antimicrobial, antiallergic, cytotoxic and antitumor activities, but still the most important action of phenolic compounds is their antioxidant potential (Chu et al, 2000; Fukumoto et al, 2000; Chi-Tai and Gow-Chin, 2006; Germano et al, 2006; Podsedek et al, 2006; Podsedek 2007).

Quantitative estimation of the total phenolic content of aqueous and methanol extracts of the selected medicinal plants was done by Folin-Ciocalteu's reagent (Zhou and $\mathrm{Yu}, 2006$ ) compared to standard gallic acid (Tab. 1, Fig. 1, 3 ) showed that there was minor difference of their quantities depending on the solvent used for extraction; the highest percent of total phenolic compounds were calculated for taqueous extracts of Coronilla scorpioides L., Forsskaolea tenacissima L., Crataegus sinaica Boiss., Pistacia khinjuk Boiss. and Loranthus acacia Benth.; they were $916.7 \pm 4.80,813.7 \pm 4.16,744.9 \pm$ $4.93,549.0 \pm 3.93 \& 460.8 \pm 4.02 \mathrm{mg} \%$ while those of methanol extracts were estimated in Coronilla scorpioides, Forsskaolea tenacissima, Crataegus sinaica, Loranthus acacia and Pistacia khinjuk; they were 915.6 44.86 , $664.6 \pm 4.16,659.3 \pm 4.80,590.8 \pm 4.49$ \& $588.0 \pm 3.85 \mathrm{mg} \%$ respectively.

The results proved that the solvents for extraction of phenolic compounds varies individually by varying medicinal plant used i.e. total phenolic content of Crataegus sinaica aqueous extract was $744.9 \pm 4.93 \mathrm{mg} \%$ while its methanol one contained $659.30 \pm 4.80$ $\mathrm{mg} \%$ whereas methanol extract of Loranthus acacia contained 590.80 \pm 4.49 $\mathrm{mg} \%$ of total phenolic compounds; aqueous one showed $460.80 \pm 4.02 \mathrm{mg} \%$.

Total phenols were measured in terms of gallic acid equivalent where the standard curve equation is $(\mathrm{y}=0.05$, $\mathrm{x} \pm 0.0545, \mathrm{r} 2=0.9873)$. 
Reactive oxygen species (ROS) such as ${ }^{\circ} \mathrm{O}_{2}$ (superoxide anion), $\mathrm{H}_{2} \mathrm{O}_{2}$ (hydrogen peroxide), and ${ }^{\circ} \mathrm{OH}$ (hydroxyl radical) may cause tissue damage, resulting from the imbalance between such reactive oxygen species generated and the natural scavenging system and seem to be implicated in the pathology of a number of disorders as atherosclerosis, ischemia-reperfusion injury, cancer, malaria, diabetes, inflammatory joint, asthma, cardiovascular diseases, cataracts, immune system decline, and could play a role in neurodegenerative diseases and aging processes (Singh, 1989; Squadrito and Pryor, 1998; Dorman et al, 2003; Young et al, 2005; De Pascual-Teresa et al, 2010).

Many researchers focused on the powerful but non-toxic antioxidants from natural sources, such natural antioxidants could prevent formation of such reactive species-related disorders in human beings instead of synthetic antioxidants that suspected of causing or prompting negative health effects and strong restrictions encountered their application with an urgent trend to substitute them with natural antioxidants (Grice, 1986; Wichi, 1988; Chu, 2000; Hosny et al, 2002).

The antioxidant potential of phenolic compounds is related to its chemical structure that confers them redox properties, they can play an important role in adsorbing and neutralizing reactive oxygen species (ROS), quenching singlet and triplet oxygen, or decomposing peroxides and so great variety of natural medicinal plants have been screened for their antioxidant activities and results have shown that the raw extracts or isolated pure compounds among them there were the more effective antioxidants in vitro compared to BHT or vitamin E (Gordon and Weng 1992; Gu and Weng 2001; Ross and Kasum, 2002; Pyo et al, 2004; Othman et al, 2007; Ali et al, 2008; Ibrahim et al, 2010; Abdel-Hady et al, 2011; El-Hela et al, 2011) and so medicinal plants are potential source of natural antioxidants (Cesquini et al, 2003).

The DPPH is a free radical which has been widely been used to test the free radical scavenging ability of various samples (Cuvelier et al, 2000; Shimoji et al, 2002; Sakanaka et al, 2005), it is a stable free radical has a characteristic absorbance at $517 \mathrm{~nm}$ and was used to study the radical-scavenging effects of the extracts as antioxidants donate protons to this radical, the absorbance decreases and so for evaluation of the DPPH scavenging effects of the extracts of the chosen plants percent DPPH inhibition was investigated.

Qualitative TLC-DPPH assay of tested extracts showed that most of them are active compounds as DPPH scavengers appearing as zones with different $R_{f}$ values at in the chromatogram, these results directed the research to quantitative estimation of the antioxidant capacity of each extract individually.

Quantitative estimation of the antioxidant potential of different extracts was done spectrophotometrically by using 
DPPH method revealed that most of the tested extracts possess significant free radical scavenging activity which is proven to be more potent when compared with the reference synthetic antioxidant standard butylated hydroxytoluene (BHT), the most significant percent free radical scavenging potentials (Tab. 1, Figs. $2 \& 3$ ) were recorded among the aqueous and methanol extracts of the Coronilla scorpioides, Forsskaolea tenacissima, Crataegus sinaica, Pistacia khinjuk and Loranthus acacia; they were $454.8 \pm 4.83$, $418.4 \pm 4.16, \quad 399.10 \pm 4.9, \quad 342.50 \pm 2.72$ and $239.7 \pm 2.91 \%$ for aqueous extracts \& 452.9 $\pm 4.94,389.6 \pm 4.6,378.48$ $\pm 3.84,352 . \pm 3.06 \& 346.5 \pm 2.98 \%$ for methanol ones respectively. Comparing the results of total phenolic contents and antioxidant potential showed a significant linear correlation, which means that phenolic compounds provide the major contribution to the antioxidant activity of these plant extracts evaluated by these assays. This is in line with similar correlations between total phenolic content and antioxidant activity of various plants was reported (Nencini et al, 2007; Abdel-Hady et al, 2011; El-Hela et al, 2011).

Mosquito-borne diseases, as malaria, filariasis and viral hemorrhagic fevers are still major public health problems in the African countries because of their tropical or subtropical climate, poor drainage system especially during the rainy seasons, and presence of many fish ponds, irrigation ditches and rice fields which provide abundant mosquito breeding places and to prevent proliferation of mosquito borne diseases and to improve quality of environment and public health, mosquito control is essential. The Yellow fever strikes an estimated 200,000 persons world-wide each year and causes an estimated 30.000 deaths (WHO, 1992), the main vector of Yellow fever is $A e$. aegyptii the most important mosquito vectors of human disease native of Africa and was introduced to the Americas in the 1600 s by the slave trade, and became highly domesticated, adapted to humans, and a highly efficient vector of epidemic yellow fever and dengue. The yellow fever virus is transmitted when a mosquito bites an infected human, then after an incubation period of 12-21 days, bites a susceptible human. A. aegyptii breeds readily in all types of the domestic and peridomestic collections of water i.e. flower vases, water drums, tin cans, broken coconut shells and even gutters (Simpson 1996; Su and Mulla, 1998).

The major tool in mosquito control operation is the application of synthetic insecticides such as organochlorine and organophosphate compounds but this has not been very successful due to human, technical, operational, ecologi$\mathrm{cal}$, and economic factors so, in recent years, the use of many of the former synthetic insecticides in mosquito control programme has been limited due to lack of novel insecticides, high cost of synthetic insecticides, concern for the environmental sustainability, harmful action on human health, and non-target populations, non-biodegradable nature, higher rate of the biological magnification through ecosystem, and increasing 
insecticide resistance on a global scale.

Before the discovery of the synthetic insecticides, natural ones such as pyrethrum, rotenone, nicotine and others have been extensively used for insect control (Balandrin, 1985; Monzon et al. 1994; Pedigo, 1996; Shaalan et al, 2005).

Some medicinal plant extracts are effective against mosquito larvae, in addition they may greatly reduce the risk of adverse ecological effects, also they do not induce pesticide resistance in mosquitoes by the virtue that these chemicals are taken from medicinal plants, they are expected to have low human toxicity and a high degree of biodegradation abilities (Choochote et al, 1999) while other researchers reviewed the efficacy of phytochemicals against mosquito larvae according to their chemical nature and described the mosquito larvicidal potential of several plant derived secondary materials, such as the essential oils, terpenes, alkaloids, steroids, isoflavonoids, pterocarpans and lignans and also documented the isolation of several bioactive toxic principles from various plants and reported their toxicity against different mosquito species (Yang et al, 2004; Shaalan et al, 2005; Kishore et al, 2011).

Searching for new control agents from natural sources has gained popularity among researchers in countries with a strong herbal tradition and large numbers of plants have been reported to possess insecticidal activity (Nagpal el al, 1996; Abdel-Hady et al, 2005; Abdel Halim, 2006; Ibrahim et al,
Brown 1986; Russell et al, 2009; 2010; Kishore et al, 2011; Kabir et al, 2013). The results gained for the screening of the Ae. aegyptii larvicidal activity (tab.1, fig. 4) proved that the Coronilla scorpioides, Forsskaolea tenacissima, Crataegus sinaica, Pistacia khinjuk and Loranthus acacia exhibited the highest potency expressed as the least $\mathrm{LC}_{50}$; the calculated values were $22.53 \pm 2.01,23.85 \pm 2.07,28.17 \pm$ $2.06,31.6 \pm 2.93 \& 39.73 \pm 4.58 \mathrm{mg} \%$ of aqueous extracts \& $18.53 \pm 1.95,18.85 \pm$ $1.67,20.17 \pm 1.85,23.28 \pm 2.70 \& 28.48$ $\pm 3.9 \mathrm{mg} \%$ of methanol extracts respectively.

By comparing the gained results of total phenolic compounds and larvicidal activity (Tab.1, Fig. 4) revealed that there was a linear correlation between larvicidal effect indicated as $\mathrm{LC}_{50}$ and total phenolic content of the investigated plants, suggesting that the phenolic compounds have significant larvicidal activity.

In Egypt, the Aedes species were encountered, Kirkpatrick (1925) reported Ae. aegyptii and Gad (1963) identified Ae. aegypti, Ae. caspius and Ae. detritus. Holstein (1967) reported complete eradication of Ae. aegypti from Egypt. Mostafa et al. (2002) reported Ae. detritus in governorates of Assiut, $\mathrm{Al}$ Fayium, Giza, Aswan, Al Wady Al Gadeed and South Sinai. Ae. caspius was found in Assiut and Aswan and as larvae in Kena and $\mathrm{Al}$ Wady $\mathrm{Al}$ Gadeed. Morsy et al. (2003, 2004) found Ae. caspius in Qalyoubia, Giza and the Greater Cairo. Shaalan et al. (2005a, b) in Aswan found Ae. aegypti in water sources. Mikhail et al. (2009) 
reported Ae. caspius and Ae. detritus in Greater Cairo, Sharkia, Qualyoubia and Giza. Abdel-Hamid et al. (2011) in El Menoufia reported Ae. (O.) caspius and Ae. (O.) detritus. Shoukry et al. (2012) in Toshka at southern Egypt identified adults and immature stages of Ae. aegypti. Undoubtedly, mosquitoes-borne diseases are threat worldwide (Mikhail et al, 2009).

Undoubtedly, re-emerging of the Ae. aegypti in Egypt mainly Aswan Governorate (Essam et al, 2006) and in Toshka Project (Heikal et al, 2011; Shoukry and Morsy, 2011; Shoukry et al, 2012; Morsy, 2012), which is the main mosquito-vector of the Yellow fever (CDC, 2010a), the Dengue and Dengue hemorrhagic fever (El-Bahnasawy et al, 2011) and Chikungunya fever (CDC, 2010b).

\section{Conclusion}

Searching for the new natural antioxidants become an urgent demand due to thehealth hazards accompanying the use of synthetic ones and the strict need of such antioxidants to decline many the health disasters caused by liberated free radicals.

Moreover, the increasing insecticide resistance require strategies to prolong the use of highly effective vector control compounds, medicinal plants can kill, deform the post-embryonic molting stages of mosquitoes could be a valuable approach in integrated vector management programs to replace synthetic pesticides. The outcome results proved that the most suitable medicinal plants used as antioxidant drugs and larvicidal agents are Coronilla scorpi- oides, Forsskaolea tenacissima, Crataegus sinaica, Pistacia khinjuk and Loranthus acacia as all shared in containing high content of total phenolic compounds.

\section{References}

Abbott, W, 1925: A method for computing the effectiveness of an insecticide. J. Econ. Entomol. 18:265-7.

Abdalmagid, MA, Alhusein, ShH, 2008: Entomological investigation of Aedes aegyptii in Kassala and Elgadarief States, Sudan, Sudanese J. Pub. Hlth, 3, 2:77-8.

Abdel Halim, AS, 2006: Efficacy of Cinnamomum zeylanicum on third stage larvae and adult fecundity of Musca domestica and Anopheles pharoensis. J. Egypt. Soc. Parasitol. 36, 1:329-34.

Abdel-Hady, NM, Abdel-Halim, SA, Al-Ghadban, AA, 2005: Chemical composition and insecticidal effect if the volatile oils of the leaves and flowers of Lantana camara L. cultivated in Egypt. J. Egypt. Soc. Parasitol. 35, 2: 687-98

Abdel-Hady, NM, Gouda, TM, ElHela, AA, Morsy, TA, 2011: Inter-relation of antioxidant, anticancer and anti-Lieshmania effects of some selected Egyptian plants and their phenolic constituents. J. Egypt. Soc. Parasitol. 41, 3:785-800

Abdel-Hamid, YM, Soliman, MI, Kenawy, MA, 2011: Geographical distribution and relative abundance of culicine mosquitoes in relation to the transmission of lymphatic filariasis in El Menoufia Governorate, Egypt. J. Egypt. Soc. Parasitol. 41, 1:109-18. 
Abudulai, MBM, Shepard, PL, Mitchell, C, 2001: Effects of neem ( $\mathrm{Aza}$ dirachta indica A. Juss) on predators of Nezara viridula (L.) (Hemiptera: Heteroptera: Pentatomidae). J. Agricult. Urban Entomol. 18:105-15.

Ali, BH, Blunden, G, Tanira, MO, Nemmar, A, 2008: Some phytochemical, pharmacological and toxicological properties of ginger (Zingiber officinale Roscoe): a review of recent research. Food Chem. Toxicol. 46:409-20.

Balandrin, M, 1985: Natural plant chemicals: sources of industrial and medicinal material. Science 228:1154-60.

Bhuwan, B, Mishra, A, Vinod, KT, 2011: Natural products in drug discovery and |investigation, Opportunity, Challenge and Scope of Natural Products in Medicinal Chemistry.

Brown, AW, 1986: Insecticide resistance in mosquitoes: A pragmatic review. J. Am. Mosq. Control Assoc. 2: 123-40.

Cai, YZ, Sun, M, Corke, H, 2003: Antioxidant activity of betalains from plants of the Amaranthaceae. J. Agricult. Food Chemist. 51, 8:2288-94.

Cavin, A, Hostettmann, K, Dyatmyko, W, Potterat, O, 1998: Antioxidant and lipohilic constituents of Tino spora crispa. Planta Med. 64:393-6.

CDC, 2010a: Fact Sheet/Yellow Fever. http://www.cdc.gov/ncidod/dvbid / yellow fever/yf_factsheet.html.

CDC, 2010b: Chikungunya Distribution \& Global Map: For information on current outbreaks, consult CDC's Travelers Health http://www.cdc.gov/travel/def-fault.aspx).
Cesquini, M, Torsoni, MA, Stoppa, GR, Ogo, SH, Booh, T, 2003: Induced oxidative damage in sickle red blood cells and the role of flavonoids. Biomed. Pharmacother. 57:124-9.

Chi-Tai, Y, Gow-Chin, Y, 2006: Induction of hepatic antioxidant enzymes by phenolic acids in rats is accompanied by increased levels of multidrug resistance-associated protein 3 mRNA expression. J. Nutrit.136:11-5.

Choochote, W, Kanjanapothi, D, Panthong, A, Taesotikul, T, Jitpakdi, A, et al, 1999: Larvicidal, adulticidal and repellent effects of Kaempferia galanga. Southeast Asian J. Trop. Med. Pub. Hlth. 30:470-6.

Chu, Y, 2000: Flavonoid content of several vegetables and their antioxidant activity. J. Sci. Food Agricul. 80:5616.

Crozier, A, Jaganath, IB, Cliford, M N. 2006: Phenols, polyphenols and tannins: An overview. In: Crozier A, Cli fford MN, Ashihaea H, Ed.Plant Secondary Metabolites, Oxford: Blackwell.

Cushnie, TPT. Lamb, AJ, 2005: Antimicrobial activity of flavonoids. Int. J. Antimicrob. Agents 26:343-56.

Cuvelier, ME, Bondet, V, Berset, C, 2000: Behavior of phenolic antioxidants (DPPH) for estimating antioxidant activity, Songklanakarin. J. Sci. Technol. 26. 2:211-9.

De Pascual-Teresa, S, Moreno, DA, Garcia-Viguera, C, 2010: Flavanols and anthocyanins in cardiovascular health: A review of current evidence. Int. J. Mol. Sci. 11:1679-703. 
Dorman, HJD, Kosar, M, Kahlos, K, Holm, Y, Hiltunen, R, 2003: Antioxidant properties and composition of aqueous extracts from Mentha species, hybrids, varieties, and cultivars. J. Agric. Food Chem. 51:4563-9.

El-Bahnasawy, MM, Khalil, HH, Morsy, AT, Morsy TA, 2011: Threat of dengue fever and dengue hemorrhagic fever to Egypt from travelers. J. Egypt. Soc. Parasitol. 41, 2:289-306.

El-Hela, A, Ibrahim, MT, Abdel-Hady, NM, Abu-Elwafa, SA, 2011: Pharmacognostical studies of Russelia equistiformis (Scrophelariaceae) cultivated in Egypt. Az. J. Pharm. Sci. 44: 267-83.

Elliott, AC, Woodward, WA, 2007: Statistical Analysis Quick Reference Guidebook: With SPSS examples. ISBN: 9781412925600.

Essam, Abdel SS, Deon, Vahid C, Bruce, B, Mohamed, Wagdy FY, Abdel Wahab, HA, Abdel Hamid, M, 2006: Efficacy of botanical extracts from Callitris glaucophylla, against Aedes aegypti and Culex annulirostris mosquitoes. Trop. Biomed. 23, 2:1805.

Finney, DJ, 1971: Probit Analysis. Cambridge: Cambridge University Press.

Fukumoto, LR, Mazza, G, 2000: Assessing antioxidant and prooxidant activities of phenolic compounds. J. Agric. Food Chem. 48:3597-604.

Gad, AM, 1963: Insects of Medical Importance. Research Institute of Medical Entomology. Ministry of Health, Dokki, Giza, Egypt.
Germano, MP, D'Angelo, VD, Biasini, T, Sanogo, R, De Pasquale, R, Catania, S, 2006: Evaluation of the antioxidant properties and bioavailability of free and bound phenolic acids from Tichilia emetic Vahl. J. Ethnopharmacol.105, 3:368-73.

Gialvez, M, Martín-Cordero, M, Peter, J, María, J, 2005: Antioxidant activity of Plantago bellardii All. Phytother. Res. 19, 12:1074-6.

Gordon, MH, Weng, XC, 1992: Antioxidant properties of extracts from tanshen (Salvia miltiorrhiza Bunge). Food Chem. 44:119-22.

Gratz, NG, 1993: Lessons of Aedes aegypti control in Thailand. Med. Vet. Entomol. 7:1-10.

Grice, HC, 1986: Safety evaluation of butylated hydroxytoluene (BHT) in the liver, lung and gastrointestinal tract. Food Chem. Toxicol. 24:1127-30.

Gu, LW, Weng, XC, 2001: Antioxidant activity and components of Salvia plebeia R.Br. a Chinese herb. Food Chem. 73:299-305.

Gubler, DJ, 1998: Dengue and dengue hemorrhagic fever. Clin. Microbiol. Rev. 11:488-96.

Halliwell, B, 1994: Free radicals, antioxidants, and human disease: curiosity, cause, or consequence? Lancet 344, 8924:569-75.

Heikal, OM, El-Bahnasawy, MM, Morsy, AYA, Khalil, HHM, 2011: Aedes aegypti re-emerging in Egypt: A review and what should be done? J. Egypt .Soc. Parasitol. 41, 3:785-814.

Holstein, M. 1967: Dynamics of $A e-$ des aegypti: Distribution, density and 
seasonal prevalence in the Mediterranean Area. Bull. Org. Mon. Santre, WHO 36:541-3.

Hosny, M, Rosazza, JPN, 2002: Novel oxidations of $(+)$ - Catechin by horseradish peroxidase and laccase. J. Agric. Food Chem. 50:S639-45.

Husham, O, Abdalmagid, MA, Brair, M 2010: Status susceptibility of dengue vector; Aedes aegyptii to different groups of insecticides in Port Sudan City, Red Sea State. Sudanese J. Pub. Hlth. 5, 4:199-202

Ibrahim, J, Mira, FY, Nazni, WA, Jamia, AJ, 2005: Insecticidal activities of the leaf oils of eight Cinnamomum species against Aedes aegypti and Aedes albopictus. Pharmacol. Biol. 43, 6:526-32.

Ibrahim, TA, El-Hefnawy, HM, EIHela, AA, 2010: Antioxidant potential and phenolic acid content of certain cucurbitaceous plants cultivated in Egypt. Nat. Product Res. 24, 16:1537-45

Jacobs, M, 2000: Dengue: emergence as a global public health problem and prospects for control. Trans. R. Soc. Trop. Med. Hyg. 94:7-8.

Kabir, KE, Choudhary, MI, Ahmed, S, Tariq, RM, 2013: Growth-disrupting, larvicidal and neurobehavioral toxicity effects of seed extract of Seseli diffusum against Aedes aegypti (L.) (Diptera: Culicidae). Ecotoxicol. Environ. Saf. 90:52-60.

Kantachuvessiri, A 2002: Dengue hemorrhagic fever in Thailand. Southeast Asian J. Trop. Med. Pub. Hlth. 33:5662.
Kirkpatrick, TW, 1925: Mosquitoes of Egypt: The Egyptian Government Press, Egypt.

Kishore, N, Mishra, BB, Tiwari, VK, Tripathi, VA, 2011: Review on natural products with mosquitosidal potentials: Opportunity, challenge and scope of natural products in medicinal chemistry. Kerala: Research Signpost.

Klocke, JA, 1989: Plant compounds as source and models of insect control agents. In: Economic and Medicinal Plant Research. Hostettmann, K. (ed) Academic, London.

McDonald, S, Prenzier, PD, Autolovich, M, Robard, K, 2001: Phenolic content and antioxidant activity of olive extracts. Food Chemist. 73:73-84.

Mikhail, MW, Al-Bursheed, KhM, AbdEl-Halim, AS, Morsy, TA, 2009: Studies on mosquito borne diseases in Egypt and Qatar. J. Egypt. Soc. Parasitol. 39, 3:745-56.

Molyneux, P, 2004: The use of the stable free radical diphenylpicrylhydrazyl (DPPH) for estimating antioxidant activity, Songklanakarin J. Sci. Technol. 26, 2:211-9.

Monzon, RB, Alvior, JP, Luczon, L L, Morales, AS, Mutuc, FE, 1994: Larvicidal potential of five Philippine plants against Aedes aegypti (Linnaeus) and Culex quinquefasciatus (Say), Southeast Asian J. Trop. Med. Public Hlth. 25:755-9

Morsy, TA, 2011: Insect bites and what is eating you? J. Egypt. Soc. Parasitol. 42, 2:291-308.

Morsy, TA, Khalil, NM, Habib, FSM, El-Laboudy, NM, 2003: Culicini 
mosquito larvae in Greater Cairo. J. Egypt. Soc. Parasitol. 33, 3:717-32.

Morsy, TA, Khalil, NM, Habib, FS M, El-Laboudy, NA, 2004: Seasonal distribution of culicini larvae in the Greater Cairo. J. Egypt. Soc. Parasitol. 34, 1:143-52.

Mostafa, AA, Allam, K, Osman, M, 2002: Mosquito species and their densities in some Egyptian governorates. J. Egypt. Soc. Parasitol. 32, 1:9-20.

Nagpal, BN, Srivastava, A, Sharma, VP, 1996: Control of mosquito breeding using scrapings treated with Neem oil. Indian J. Malariol. 32:64-9

Nencini, C, Cavallo, F, Capasso, A, Franchi, GG, Giorgio, G, Micheli, L, 2007: Evaluation of antioxidative properties of Allium species growing wild in Italy. J. Phytother. Res., 2:874-8.

Niki, E 1997: Free radicals, antioxidants, and cancer. In: Food Factors for Cancer Prevention. Ohigashi, H, Osawa, T, Terao, J., Watanabe, S, Yoshikawa, T, (Eds.), Springer, Tokyo.

Nurmikko, TJ, Serpell, MG, Hoggart, B, Toomey, PJ, Morlion, BJ, et al, 2007: Epidemics and Research in the last forty years and prospects for the extracts of Japanese persimmon leaf tea (kakinoha-cha). Food Chemist. 89, 4:133-210.

Othman, A, Ismail, A, Ghani, NA, Adenan, I, 2007: Antioxidant capacity and phenolic content of cocoa beans. Food Chem. 100, 1523-1530.

Owen, RW, Giacosa, A, Hull, WE, Haubner, R, Spiegelhalder, B, Bartsch, H, 2000: The antioxdant/ anticancer potential of phenolic compou- nds isolated from olive oil. Euro J. Cancer, 36, 10:1235-47.

Pancharoen, C, Kulwichit, W, Tantawichien, T, Thisyakorn, U, Thisyakorn, C 2002: Dengue infection: A global concern. J. Med. Assoc. Thailand 85:25-33.

Pedigo LP, 1996: Entomology and Pest Management. New Jersey: Prentice-Hall.

Pereira, DM, Valentao, P, Pereira, J A, Andrade, PB, 2009: Phenolics: From chemistry to biology. Molecules, 14:2202-11.

Podsedek, A, 2007: Natural antioxidants and antioxidant capacity of Brassica vegetables: A review. Food Sci. Technol. 40:1-11.

Podsedek, A, Sosnowska, D, Redzynia, M, Anders, B, 2006: Antioxidant capacity and content of Brassica oleracea dietary antioxidants. Int. J. Food Sci. Technol. 41:49-58.

Poulson, HE, Prieme, H, Loft, S, 1998: Role of oxidative DNA damage in cancer initiation and promotion. Euro J. Cancer Prevent. 7, 1:9-16.

Pyo, YH, Lee, TC, Logendrac, L, Rosen, RT, 2006: Antioxidant Activity and Phenolic Plant Secondary Metabolites: Occurrence, Structure and Role in the Human Diet Eds.; Blackwell: Oxford, the United Kingdom.

Ross, JA, Kasum, CM, 2002: Dietary flavonoids: Bioavailability, metabolic effects, and safety. Ann. Rev. Nutrit. 22:19-34.

Russell, TL, Kay, BH, Skilleter, GA, 2009: The environmental effects of the 
mosquito insecticides on saltmarsh invertebrate fauna. Aquat. Biol. 6:7790

Sakanaka, S, Tachibana, Y, Okada, Y, 2005: Preparation and antioxidant proper-ties of extract of Japanese persimmon leaf (kakinoha-cha), Food Chem., 46:569-75.

Sala, A, Recio, MD, Giner, RM, Manez, S, Tournier, H, Schinella, G, Rios, JL, 2002: Antiinflammatory and antioxidant properties of Helichrysum italicum. J. Pharmacy Pharmacol. 54: 365-71.

Schaafsma, AW, 1990: Resistance to malathion in populations of Indian meal moth, Plodia interpunctella (Lepidoptera: Pyralidae). Proceed. Entomol. Soc. Ontario, 121:101-14.

Shaalan, EA, Canyon, DV, Younes, MW, Abdel-Wahab, H, Mansour, AH, 2005a: Synergistic efficacy of botanical blends with and without synthetic insecticides against Aedes aegypti and Culex annulirostris mosquitoes. J. Vector Ecol. 30, 2:284-8.

Shaalan, EA, Canyon, DV, Younes, MW, Abdel-Wahab, H, Mansour, A H, 2005b: Effects of sub-lethal concentrations of synthetic insecticides and Callitris glaucophylla extracts on the development of Aedes aegypti. J. Vector Ecol. 30, 2: 295-8.

Shaalan, EA, Canyonb, DV, Younes, MW, Abdel-Wahab H, Mansoura AH, 2005c: A review of botanical phytochemicals with mosquitocidal potential. Environ. Int. 3:1149-66.

Shimoji, Y, Tamura, Y, Nakamura, Y, Nanda, K, Nishidai, S, Nishikawa,
Y, 2002: Isolation and identification of DPPH radical scavenging compounds in kurosu (Japanese unpolished rice vinegar). J. Agric. Food Chem. 50: 6501-3.

Shoukry, NM, Morsy, TA, 2011: Arthropod borne diseases at Toshka, Upper Egypt. World J. Zool. 6, 2:12633.

Shoukry, NM, Elwan, MA, Morsy' T A, 2012: Aedes aegypti (Linnaeus) reemerging in southern Egypt. J. Egypt. Soc. Parasitol. 42, 1:41-50

Simpson, DIH, 1996: Arbovirus infections. In: Manson's Tropical Diseases. Bath, UK: Saunders.

Singh, A, 1989: Physicochemical and physiological aspects. In: Handbook of Free Radicals and Antioxidants in Biomedicine. Vol. 1, CRC Press Inc.: Boca Raton, Florida, USA.

Singleton, VL, Rossi, JA, 1965: Colourimetry of total phenolics with phospho-molybdic phosphotungstic acid reagents, Am. J. Encol. Vitic.16:14458.

Squadrito, GL, Pryor, WA, 1998: Oxidative chemistry of nitric oxide: the roles of superoxide, peroxynitrite, and carbon dioxide. Free Rad. Biol. Med. 25, 4/5:392-403.

Su, T, Mulla, MS, 1998: Ovicidal activity of Neem products (Azadirachtin) against Culex tarsalis and Culex quinquefasciatus (Diptera: Culicidae). J. Am. Mosq. Control Assoc.14:204-9.

Sun, J, Chu, YF, Wu, XZ, Liu, RH, 2002: Antioxidant and antiproliferative activities of common fruits. J. Agricult. Food Chemist. 50, 25:7449-54. 
e activities of common fruits. J. Agricul. Food Chemist. 50, 25:7449-54.

Swaddiwudhipong, WP, Lerdlukanavonge, PK, Koonchote, P, Chaovakiratipong, C, 1992: A survey of knowledge, attitude and practice of the prevention of dengue hemorrhagic fever in an urban community of Thailand. Southeast Asian J. Trop. Med. Pub. Hlth. 23:207-11.

WHO 1992: Division of Epidemiological Surveillance and Health Situation and Trend Assessment, Global Health Situation and Projections: Estimates. Geneva, Switzerland. WHO, 1996: Report of the WHO informal consultation on the evaluation and testing of insecticides, Geneva.

Wichi, HP, 1988: Enhanced Tumor development by butylated hydroxyanisole (BHA) from the perspective of effect on fore stomach and oesophageal squamous epithelium. Toxicol. 26:71723.
Yang, CS, Landau, JM, Huang, MT, Newmark, HL, 2001: Inhibition of carcinogenesis by dietary polyphenolic compounds. Ann. Rev. Nutrit. 21:381406.

Yang, Y, Park, I, Kim, E, Lee, H, Ahn, Y, 2004: Larvicidal activity of medicinal plant extracts against Aedes aegypti, Ochlerotatus togoi and Culex pipiens pallens (Diptera: Culicidae). J. Asia Pacif. Entomol. 7, 2:227-32.

Young, JE, Zhao, X, Carey, EE, WeIti, R, Yang, SS, Wang, WQ, 2005: Phytochemical phenolics in organically grown vegetables. Mol. Nutrit. Food Res. 49:1136-42.

Zheng, W, Wang, SY, 2001: Antioxidant activity and phenolic compounds in selected herbs. J. Agricult. Food Chemist. 49, 11: 5165-70.

Zhou, K, Yu, L, 2006: Total phenolic contents and antioxidant properties of commonly consumed vegetables grown in Colorado. Lebensmittel-Wissenschaft Technol., 39, 10:1155-62. 
Fig. 3: Total phenolic compounds content and antioxidant potential of top five potent medicinal plants:

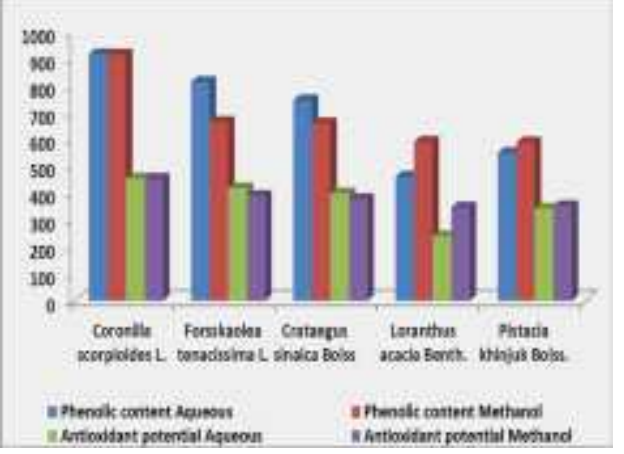

Fig. 4: Aedes aegyptii larvicidal activity of the top five potent medicinal plants:

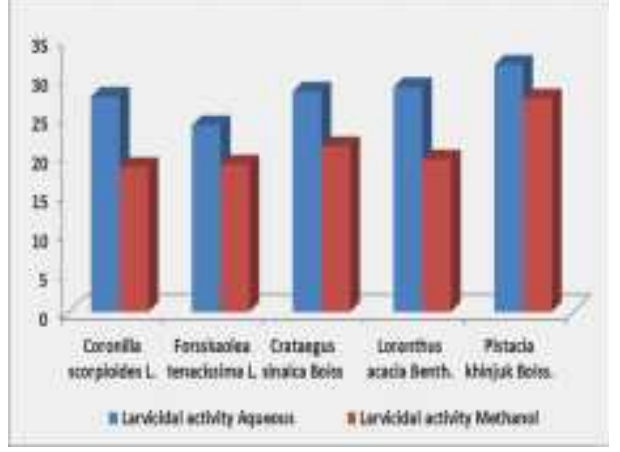

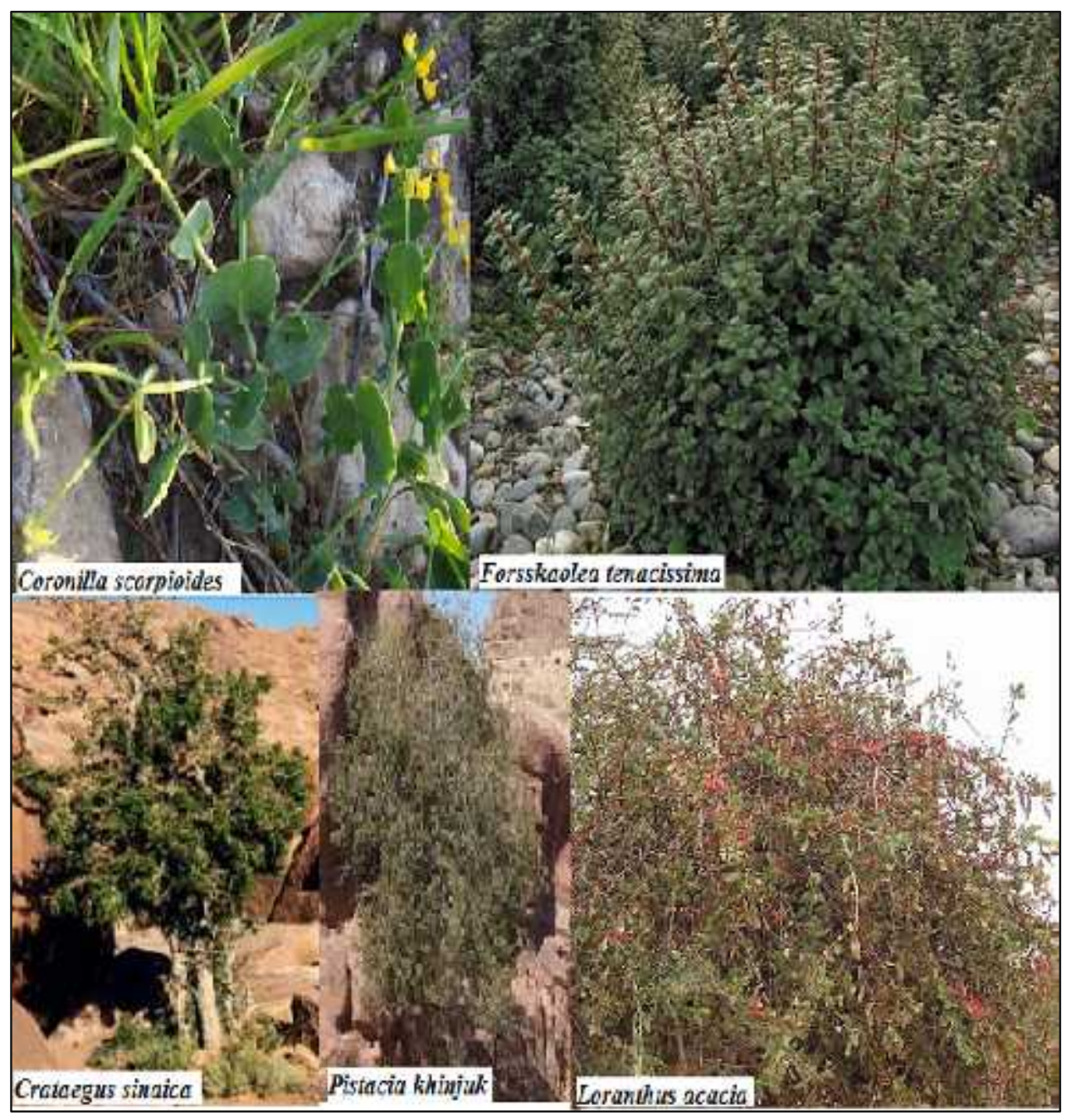

